\title{
Structure demonstration of perovskite oxide and its epitaxial thin films by second harmonic generation
}

\author{
WANG JieSu ${ }^{1}$, GE Chen ${ }^{1,2}$, GUO Er-Jia ${ }^{1,2}$, XU XiuLai ${ }^{1,2,3}$, WANG Can ${ }^{1,2,3}$ \& JIN KuiJuan ${ }^{1,2,3^{*}}$ \\ ${ }^{1}$ Institute of Physics, Chinese Academy of Sciences, Beijing 100190, China; \\ ${ }^{2}$ University of Chinese Academy of Sciences, Beijing 100049, China; \\ ${ }^{3}$ Songshan Lake Materials Laboratory, Dongguan 523808, China
}

Received November 2, 2019; accepted November 19, 2019; published online November 26, 2019

\begin{abstract}
Citation: Wang J S, Ge C, Guo E J, et al. Structure demonstration of perovskite oxide and its epitaxial thin films by second harmonic generation. Sci China Tech Sci, 2020, 63: 874-876, https://doi.org/10.1007/s11431-019-1484-3
\end{abstract}

Perovskite oxide materials and their epitaxial thin films have attracted wide attention in various research fields, due to the new properties discovered at every period. For the epitaxial perovskite oxide thin films, the interaction between the films and substrates could bring in some novel physical properties, such as two-dimensional electron gas [1], positive colossal magnetoresistance [2], polar metal state [3,4], interfacial enhancement effect [5-7]. The (dis)appearance and regulation of these properties are usually accompanied with structural distortion changing of films, which may originate from the thickness variation, substrate replacement, or oxygen vacancies [8]. Thus, it is crucial to define what structure it is and figure out how the structure evolves.

On the other hand, as the simplest example of nonlinear optic process, second harmonic generation ( $\mathrm{SHG}$ ) is a powerful tool to explore the symmetry of noncentrosymmetric structures [9-12]. It works especially well in terms of surfaces and interfaces where the different media on each side break the inversion symmetry [13], as well as the crystals and films with polar structure [14]. Resent researches have proved that SHG can be a convenient, noninvasive, and effective way to demonstrate the structure of perovskite oxide and its epitaxial thin films.

As a multiferroic material, $\mathrm{BiFeO}_{3}(\mathrm{BFO})$ and $\mathrm{BFO}$ thin films have drawn considerable attention for the potential usage in microelectronic information storage devices [15-

*Corresponding author (email: kjjin@iphy.ac.cn)
18]. When deposited on substrates, BFO thin films can exhibit different structural phases depending on the lattice mismatch and thermal expansion, and even different conclusions were obtained because of different test methods $[19,20]$. By engaging SHG technique to explore the phaserelated symmetry evolution of a series of BFO thin films with different thicknesses, it is clearly demonstrated that the structure of BFO thin films varies from tetragonal-like ( $T$ like) phase to rhombohedral-like ( $R$-like) one [9].

High quality BFO films with the thicknesses of 4, 12, 60, 100 and $180 \mathrm{~nm}$ were epitaxially grown on (001)-oriented $\mathrm{Nb}$-doped $\mathrm{SrTiO}_{3}$ (SNTO) substrates. As shown in Figure 1 (a), for ultrathin BFO films in a compressive strain state, the polarization resolved SHG pattern in $s$-out configuration processes a four-fold rotational symmetry associated with the absence of in-plane anisotropy structure, which indicates the $4 \mathrm{~mm}$ point group and the $T$-like phase of the films. This feature was kept for thicker BFO films with the thickness up to $60 \mathrm{~nm}$. For 100- and 180-nm-thick BFO films, the corresponding four-fold rotational symmetry in their patterns disappear, shown as Figure 1(b) and (c) respectively. Theoretical analysis further proved the point group are not $4 \mathrm{~mm}$ anymore but $m$, and these BFO films are in $R$-like phase. By analyzing SHG results, it is concluded that the disappearance of four-fold rotational symmetry in SHG $s$-out pattern implies the appearance of $R$-phase in epitaxial BFO thin films $[9,21]$.

Another suitable situation for using SHG to explore the 

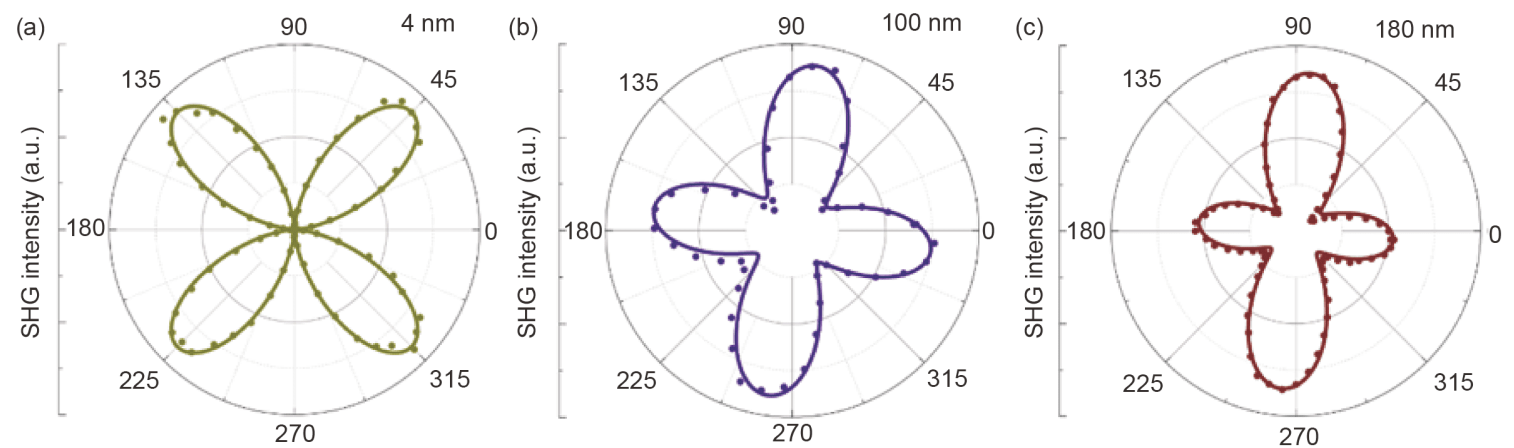

Figure 1 (Color online) Polarization resolved SHG patterns in s-out configuration for BFO films with the thickness of (a) $4 \mathrm{~nm}$, (b) $100 \mathrm{~nm}$, and (c) $180 \mathrm{~nm}$. The dots show the experimental data and the solid line are theoretical simulation results [9]. Reproduced from Sci Rep with permission.

structure evolution is the temperature dependent phase transition. More and more experimental evidences show the coexistence of displacive and order-disorder dynamics during the phase transition process of $\mathrm{BaTiO}_{3}$ (BTO). By applying SHG in this process, an initial state determined phase transition was discovered [12]. The normalized SHG intensity variation with temperature for polarized (001) and non-polarized (100) BTO crystals are quite different, as shown in Figure 2. Comparing their polarization resolved SHG patterns during the phase transition process, which indicates the corresponding structure variation, it is concluded that the dominated kinetic mechanism of phase transition around $T_{\mathrm{C}}$ is displacive for the polarized (001) BTO, while displacive and order-disorder for the non-polarized (100) BTO [12]. Moreover, by introducing SHG, it is proved the polarization structure evolution depending on temperature of $\mathrm{BTO} / \mathrm{La}_{0.5} \mathrm{Ca}_{0.5} \mathrm{MnO}_{3} / \mathrm{SrTiO}_{3}(\mathrm{BTO} / \mathrm{LCMO} /$ $\mathrm{STO}$ ) heterostructures is nonlinear due to the appearance of phase transition [22].

Besides the lattice structure evolution, SHG can also be used to illustrate the polarization state. The SH signal intensity generated only from the surfaces and interfaces are quite small compared with that from the polarization structures [23]. For polar metal, in which the traditional method of proving the existence of polarization have failed, like utilizing electric field, SHG is a powerful tool to illustrating the polarization structure. Recent researches on the promising candidate for polar metal, such as Nb-doped $\mathrm{PbTiO}_{3}$ films and $\mathrm{BTO} / \mathrm{SrTiO}_{3} / \mathrm{LaTiO}_{3}$ superlattices, have further confirmed that SHG technology is suitable in determining the polar properties in polar metals [23-25].

In addition to the intensity of SHG associated with the polarization, the relationship between SHG anisotropy patterns and the direction of polarization in perovskite ferroelectric materials have also been established. We systematically investigated the evolution of polarization resolved SHG patterns of BTO crystal at different azimuth angles, which means the net polarization direction are also different. The results illustrated that there is a linear re-

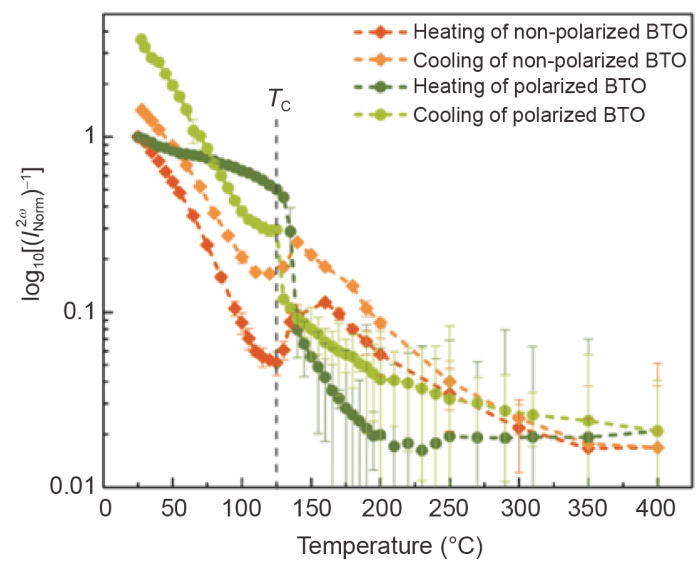

Figure 2 (Color online) Temperature dependent normalized SHG intensity variation for the polarized and non-polarized BTO crystals. The dots represent experimental data and the dash lines are for eye-guide. The grey dashed line denotes the Curie temperature $T_{\mathrm{C}}$ of $\mathrm{BTO}$ at $120^{\circ} \mathrm{C}$ [12]. Reproduced from Appl Phys Lett, with the permission of AIP Publishing.

lationship between the rotation angle of SHG patterns and the polarization angle of BTO crystals. Thus, the polarization direction of BTO crystal, even of other ferroelectric materials, can be qualitatively identified in $0^{\circ}-180^{\circ}$ by SHG technology [11].

With the popularity of lasers in various research fields, SHG technology, as an optical method to detecting noncentral symmetrical structures, has drawn more and more attention for its high sensitivity, easy construction, and nonintrusive measurement. Some recent researches related to SHG measurements especially on perovskite oxide and its thin films are given above. Actually, SHG is highly recommended in all researches involving structure variation. Further development and utilization of SHG technology is awfully expected.

This work was supported by the National Key Basic Research Program of China (Grant No. 2017YFA0303604), the National Natural Science Foundation of China (Grant Nos. 11721404, 51761145104, 11974390 and 11674385), the Key Research Program of Frontier Sciences of the Chinese Academy of Sciences (Grant No. QYZDJ-SSW-SLHO20), the Youth In- 
novation Promotion Association of CAS (Grant No. 2018008), and Postdoctoral Science Foundation of China (Grant No. 2019M650877).

1 Ohtomo A, Hwang H Y. A high-mobility electron gas at the $\mathrm{LaAlO}_{3}$ / $\mathrm{SrTiO}_{3}$ heterointerface. Nature, 2004, 427: 423-426

2 Jin K, Lu H, Zhou Q, et al. Positive colossal magnetoresistance from interface effect in p-n junction of $\mathrm{La}_{0.9} \mathrm{Sr}_{0.1} \mathrm{MnO}_{3}$ and $\mathrm{SrNb}_{0.01} \mathrm{Ti}_{0.99} \mathrm{O}_{3}$. Phys Rev B, 2005, 71: 184428

$3 \mathrm{He} \mathrm{X}$, Jin K. Persistence of polar distortion with electron doping in lone-pair driven ferroelectrics. Phys Rev B, 2016, 94: 224107

4 Ma C, Jin K J. Design strategy for ferroelectric-based polar metals with dimensionality-tunable electronic states. Sci China-Phys Mech Astron, 2018, 61: 097011

5 Malik I A, Ke X X, Liu X, et al. Solid state reaction for the formation of spinel $\mathrm{MgFe}_{2} \mathrm{O}_{4}$ across perovskite oxide interface. Sci China-Phys Mech Astron, 2017, 60: 097721

6 Zhao R Q, Jin K J, Guo H Z, et al. A study on surface symmetry and interfacial enhancement of $\mathrm{SrTiO}_{3}$ by second harmonic generation. Sci China-Phys Mech Astron, 2013, 56: 2370-2376

7 Jin K, Lu H, Zhao K, et al. Novel multifunctional properties induced by interface effects in perovskite oxide heterostructures. Adv Mater, 2009, 21: 4636-4640

8 Wan Q, Jin K J, Li Q Q, et al. Oxygen vacancies effects on phase diagram of epitaxial $\mathrm{La}_{1-x} \mathrm{Sr}_{x} \mathrm{MnO}_{3}$ thin films. Sci China-Phys Mech Astron, 2017, 60: 057711

9 Wang J S, Jin K J, Guo H Z, et al. Evolution of structural distortion in $\mathrm{BiFeO}_{3}$ thin films probed by second-harmonic generation. Sci Rep, 2016, 6: 38268

10 Pavlov V V, Kalashnikova A M, Pisarev R V, et al. Magnetic-fieldinduced second-harmonic generation in semiconductor GaAs. Phys Rev Lett, 2005, 94: 157404

11 Wang J S, Jin K J, Gu J X, et al. Direct evidence of correlation between the second harmonic generation anisotropy patterns and the polarization orientation of perovskite ferroelectric. Sci Rep, 2017, 7: 9051

12 Wang J, Jin K, Yao H, et al. Temperature-dependent phase transition in barium titanate crystals probed by second harmonic generation. Appl
Phys Lett, 2018, 112: 102904

13 Rudnick J, Stern E A. Second-harmonic radiation from metal surfaces. Phys Rev B, 1971, 4: 4274-4290

14 Denev S A, Lummen T T A, Barnes E, et al. Probing ferroelectrics using optical second harmonic generation. J Am Ceram Soc, 2011, 94 : 2699-2727

15 Tian S L, Wang C, Zhou Y, et al. Effect of mechanical force on domain switching in BiFeO3 ultrathin films. Sci China-Phys Mech Astron, 2020, 63: 217711

16 Feng Y, Wang C, Tian S L, et al. Effects of $\mathrm{BaTiO}_{3}$ and $\mathrm{SrTiO}_{3}$ as the buffer layers of epitaxial $\mathrm{BiFeO}_{3}$ thin films. Sci China-Phys Mech Astron, 2017, 60: 067711

17 Choi T, Lee S, Choi Y J, et al. Switchable ferroelectric diode and photovoltaic effect in $\mathrm{BiFeO}_{3}$. Science, 2009, 324: 63-66

18 Wan Q, Jin K J, Wang J S, et al. Modulation of ultrafast laser-induced magnetization precession in $\mathrm{BiFeO}_{3}$-coated $\mathrm{La}_{0.67} \mathrm{Sr}_{0.33} \mathrm{MnO}_{3}$ thin films. Sci China-Phys Mech Astron, 2017, 60: 047511

19 Béa H, Bibes M, Petit S, et al. Structural distortion and magnetism of $\mathrm{BiFeO}_{3}$ epitaxial thin films: A Raman spectroscopy and neutron diffraction study. Phil Mag Lett, 2007, 87: 165-174

20 Rault J E, Ren W, Prosandeev S, et al. Thickness-dependent polarization of strained $\mathrm{BiFeO}_{3}$ films with constant tetragonality. Phys Rev Lett, 2012, 109: 267601

21 Tian S, Wang C, Zhou Y, et al. Manipulating the ferroelectric domain states and structural distortion in epitaxial $\mathrm{BiFeO}_{3}$ ultrathin films via Bi nonstoichiometry. ACS Appl Mater Interfaces, 2018, 10: 4379243801

22 Wang L F, Luo Y, Wang J S, et al. Temperature-dependent evolution of surface charge screening and polarization at ferroelectric surfaces. Sci China-Phys Mech Astron, 2019, 62: 987721

23 Wang J, Yao H, Jin K, et al. Magnetoresistance in metallic ferroelectrics. ACS Appl Electron Mater, 2019, 1: 1225-1232

24 Cao Y, Wang Z, Park S Y, et al. Artificial two-dimensional polar metal at room temperature. Nat Commun, 2018, 9: 1547

25 Yao $\mathrm{H}$, Wang J, Jin $\mathrm{K}$, et al. Multiferroic Metal- $\mathrm{PbNb}_{0.12} \mathrm{Ti}_{0.88} \mathrm{O}_{3-\delta}$ films on Nb-doped STO. ACS Appl Electron Mater, 2019, 1: 21092115 